\title{
Advances in Fibrolamellar Hepatocellular Carcinoma: A Review
}

\author{
Irene Isabel P. Lim ${ }^{1} \quad$ Benjamin A. Farber ${ }^{1} \quad$ Michael P. LaQuaglia $^{1}$ \\ ${ }^{1}$ Department of Surgery, Pediatric Surgical Service, Memorial Sloan \\ Kettering Cancer Center, New York, New York, United States \\ Eur J Pediatr Surg 2014;24:461-466. \\ Address for correspondence Michael P. La Quaglia, MD, FACS, FRCS \\ (Ed Hon), FAAP, Department of Surgery, Pediatric Surgical Service, \\ Memorial Sloan Kettering Cancer Center, 1275 York Avenue, Suite H-1315, \\ New York, NY 10065, United States (e-mail: laquaglm@mskcc.org).
}

\begin{abstract}
Fibrolamellar hepatocellular carcinoma is a rare primary liver tumor that often arises in the absence of cirrhosis or viral hepatitis. Compared with hepatocellular carcinoma, patients are typically younger with less comorbidities. Diagnosis is often multimodal and requires a high level of suspicion, as traditional liver pathology markers, such as serum $\alpha$ fetoprotein and transaminases, are often normal. Overall, patients respond well to surgical resection but recurrences are frequent, and alternative therapies, such as chemotherapy and radiation, are not well studied. Currently, there are no established chemotherapy regimens; there are only limited case reports of select agents, such as 5 -fluorouracil with interferon- $\alpha$ and gemcitabine with oxaliplatin, used with varying degrees of success. Because little is known about this rare tumor, the development of serum markers and alternative therapies continues to be a challenge. A major

Keywords

- liver tumors

- fibrolamellar hepatocellular carcinoma

- liver resection advancement in the understanding of this rare disease is the discovery of a functional chimeric transcript incorporating DNAJB1 and PRKACA. This finding may finally provide the basis for specific diagnostic markers and chemotherapies that patients with this disease have long needed. Here, we present advances in the surgical treatment of fibrolamellar hepatocellular carcinoma, as well as recent data on its tumor biology and pathogenesis.
\end{abstract}

\section{Introduction}

Fibrolamellar hepatocellular carcinoma (FL-HCC) is a rare primary liver tumor that was first described by Edmondson in $1956 .{ }^{1}$ Microscopically, FL-HCC is characterized by large polygonal, well-differentiated tumor cells with an eosinophilic cytoplasm interspersed with lamellar bands of collagen fibers ${ }^{2,3}$ ( - Fig. 1). Initially thought to be a variant of hepatocellular carcinoma (HCC), FL-HCC is now considered an entirely different entity: it is associated with fewer chromosomal changes and genomic heterogeneity in comparison to HCC, as well as the absence of mutations in key genes such as p53, and low levels of promoter methylation. ${ }^{4}$ It occurs in the absence of underlying cirrhosis or viral

received

October 20, 2014

accepted

October 24, 2014

published online

December 8, 2014 hepatitis. Moreover, markers such as liver enzymes and serum $\alpha$ fetoprotein (AFP) that are typically elevated in conventional HCC are within normal limits or only mildly elevated in FL-HCC. ${ }^{5}$

Accounting for less than $1 \%$ of all primary liver cancer cases, ${ }^{6}$ FL-HCC is seen largely as a pediatric and adolescent disease. $^{2,7}$ Recent studies, however, have found two agespecific incidence peaks from 10 to 30 years and 70 to 79 years of age. ${ }^{8}$ Incidence rates were found to be homogeneous across racial and ethnic groups, compared with conventional HCC, with male-to-female ratio of 1.7:1 (compared with 3.2:1 in HCC). ${ }^{8}$ While FL-HCC is more likely to be diagnosed at advanced stages with larger tumor sizes, ${ }^{9}$ patients are more likely to receive potentially curative therapy. 8,10

(c) 2014 Georg Thieme Verlag KG Stuttgart · New York
DOI http://dx.doi.org/ 10.1055/s-0034-1396420. ISSN 0939-7248. 


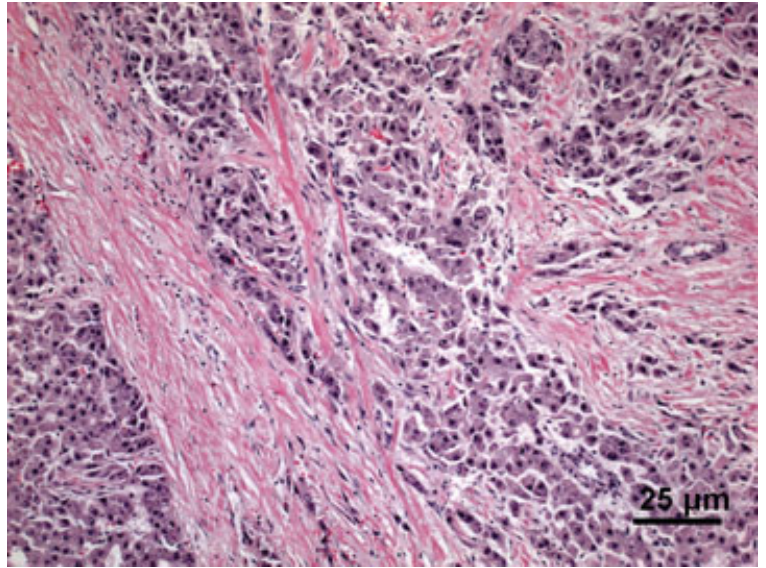

Fig. 1 Collagen bands are seen interspersed between large nests of oncocytic tumor cells in this hematoxylin and eosin stain image.

This strategy may be due in part to the younger age of the patients, as well as the lack of cirrhosis and other comorbidities. The perception that this tumor has an indolent clinical course, and that there is little proven alternative effective therapy, has encouraged an aggressive surgical approach including both resection and transplantation. ${ }^{4}$ As treatment approaches for FL-HCC remain largely surgical, this review will focus on the recent advances in surgical treatments and understanding the biology of the disease.

\section{Pathogenesis}

From its first description in $1956,{ }^{1}$ FL-HCC and its pathogenesis have yet to be definitively determined. Histologically, FL-HCC tumor cells appear to be hepatocytes and immunohistochemical studies confirm hepatocyte differentiation by staining positive for heppar-1 and glypican-3. ${ }^{11}$ FL-HCC tumor cells were also found to have markers of biliary differentiation such as CK19 and EpCAM. ${ }^{11,12}$ Recent transcriptional profiling of FL-HCC, however, has questioned its presumed hepatocyte origin. Malouf et al found overexpression of the neuroendocrine genes prohormone convertase 1 , neurotensin and calcitonin, suggesting a different origin entirely. ${ }^{13}$ Indeed, high aromatase activity and elevated estradiol levels have been noted in case reports, ${ }^{14}$ but with limited evidence to the contrary, FL-HCC is still considered to be derived from hepatocytes.

Multiple studies have attempted to explain the pathogenesis of FL-HCC. Sulaiman et al describe a patient presenting with recurrent nonhepatic hyperammonemic encephalopathy, suggesting that FL-HCC can mimic ornithine transcarbamylase deficiency. ${ }^{15}$ Riehle et al have studied the role of mammalian target of rapamycin complex 1 (mTORC1) and fibroblast growth factor receptor 1 (FGFR1) signaling in the setting of FL-HCC. ${ }^{16}$ mTORC1 activation and FGFR1 overexpression have been found in the subset of FL-HCC studied, implicating a potential role of the mTORC1 pathway in the pathogenesis of the disease. ${ }^{16}$ Advances in whole genome sequencing have revealed what may be the closest explanation for the pathogenesis of
FL-HCC. In all 15 patient samples, a functional chimera of the first exon of $D N A J B 1$, encoding a member of the heatshock 40 protein family and PRKACA, the gene encoding the cAMP-dependent protein kinase A catalytic subunit $\alpha$, was found in all tumor tissues, and absent in normal tissues. ${ }^{17}$ This chimera (-Fig. 2) appears to be responsible for both increased activity and levels of PRKACA in tissue (-Fig. 3 ). Subsequent analysis by other groups show the same chimera in additional FL-HCC samples. ${ }^{18}$ Preliminary data suggest that the chimera plays a transformative role, ${ }^{18}$ though much remains to be investigated.

Without a clear mechanism of the disease, the development of serum markers for early detection of primary, or even recurrent, FL-HCC is difficult. Nonetheless, studies have shown promising candidates such as vitamin B12, high serum vitamin B12-binding capacity, and neurotensin. ${ }^{19-21}$ Elevations in these markers have been described, but not consistently. ${ }^{5,21}$ AFP serum elevations, commonly observed in conventional HCC, are not typically seen in FL-HCC. ${ }^{5,22}$ Alternatively, FL-HCC has been associated with higher levels of expression of epidermal growth factor receptor (EGFR) compared with conventional HCC. ${ }^{23}$ Currently, definitive diagnosis is limited to the tumor's distinct appearance on microscopy, specifically the large nests of oncocytic tumor cells within fibrous lamellar collagen bands ( - Fig. 1 ).

\section{Clinical Presentation, Work-up, and Prognosis}

Clinical presentation of FL-HCC is usually nonspecific and may present with abdominal pain, malaise, and weight loss. ${ }^{4,24}$ Thus, imaging studies are an integral part of diagnosis. Multimodal imaging is utilized, particularly in the pediatric population, beginning with abdominal ultrasound followed by abdominal magnetic resonance imaging (MRI) or computed tomography (CT) scan. CT and MRI are the preferred methodologies for tumor, nodal, and systemic metastasis (TNM) staging. ${ }^{25}$ On imaging, FL-HCC tends to present as a large, heterogeneous enhancing mass (-Figs. 4 and 5) that may contain a central scar and/or calcifications. ${ }^{24,26,27}$ The value of positron emission tomography (PET) scan for conventional HCC is limited because the sensitivity of PET imaging is not sufficiently high. ${ }^{28}$ However, case reports have described unequivocal tracer uptake in FL-HCC, and further study of its role in assisting diagnosis primary or recurrent FL-HCC may be warranted. ${ }^{29}$

The prognosis for FL-HCC has traditionally been shown to be better than conventional HCC. In a recent Surveillance, Epidemiology and End Results (SEER) study encompassing the largest group of FL-HCC patients in the United States, ${ }^{8}$ FL-HCC patients between ages 0 and 39 years presented with larger tumors than patients with conventional HCC of the same ages, but 5-year relative survival rates were higher in patients with FL-HCC (40.3\% vs. 25\%). Overall 5-year survival rates irrespective of tumor size or age were similarly more favorable for FL-HCC patients compared with conventional 


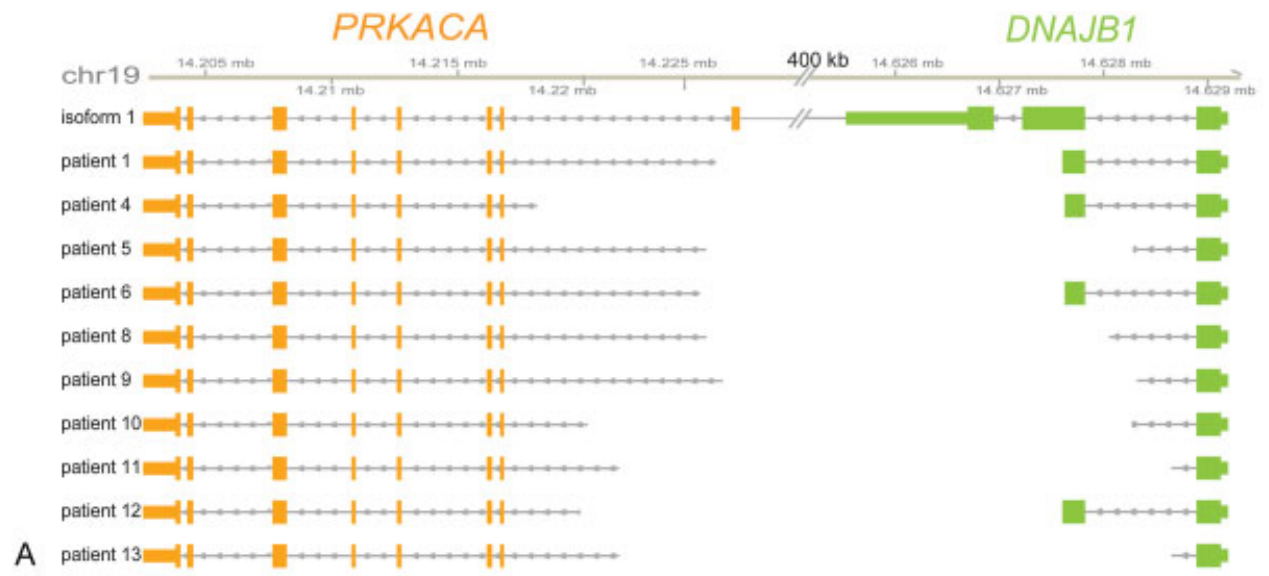

\section{Patient 1}

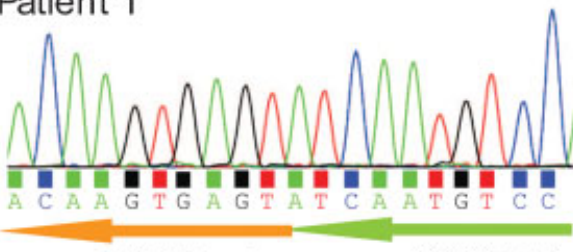

PRKACA intron 1

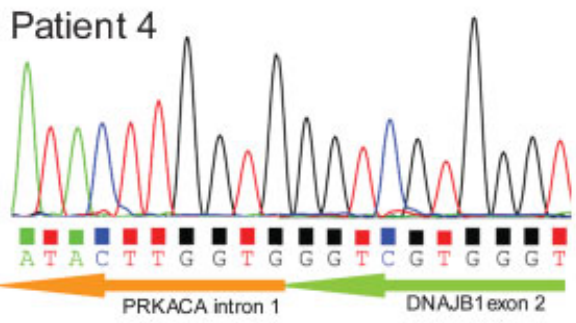

Patient 5

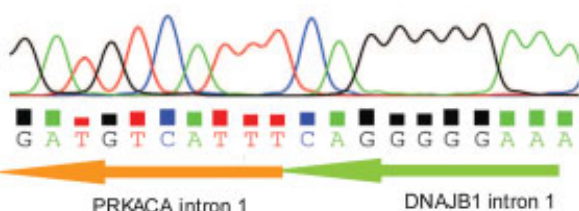

Patient 6

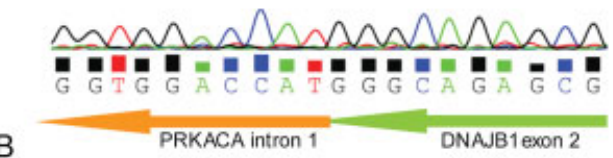

Patient 8



Patient 9

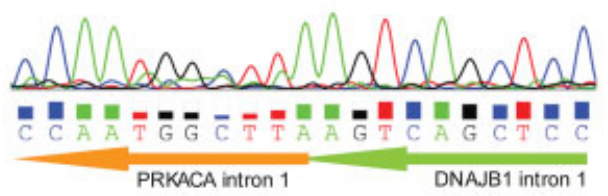

Patient 12

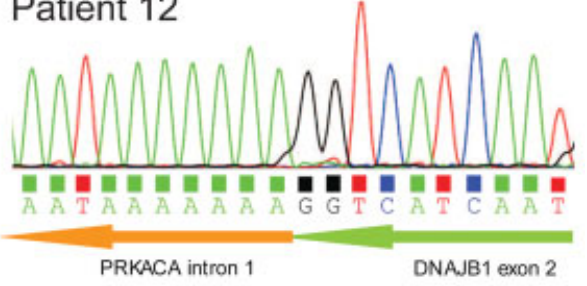

Patient 13

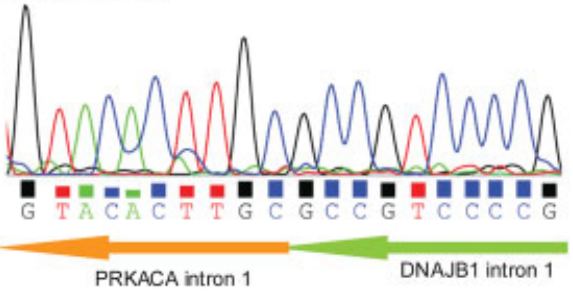

Fig. 2 DNA sequence analysis mapping the size and location of breaks in DNA between DNAJB1 and PRKACA genes to form a DNAJB1-PRKACA chimera. ${ }^{17}$ Originally published in: Honeyman JN et al. Detection of a recurrent DNAJB1-PRKACA chimeric transcript in fibrolamellar hepatocellular carcinoma. Science 2014; 343:1011. (c) 2014 American Association for the Advancement of Science. Reprinted with permission.

HCC (33.6\% vs. $16.1 \%){ }^{8}$ Long-term overall survival between FL-HCC and HCC, however, was found to be similar in two separate studies. ${ }^{30,31}$ This discrepancy may be attributable to the inclusion of adult patients in the SEER data, while the studies showing similarity in survival rates were limited to the pediatric and adolescent population. Relative survival rates may be longer in FL-HCC compared with HCC due to late recurrences that are common in FL-HCC. ${ }^{30}$ Regardless, poorer outcomes and decreased survival rates have consistently been described in patients with extrahepatic disease, such as positive regional lymph nodes and distant metastatic disease. $^{32}$ 


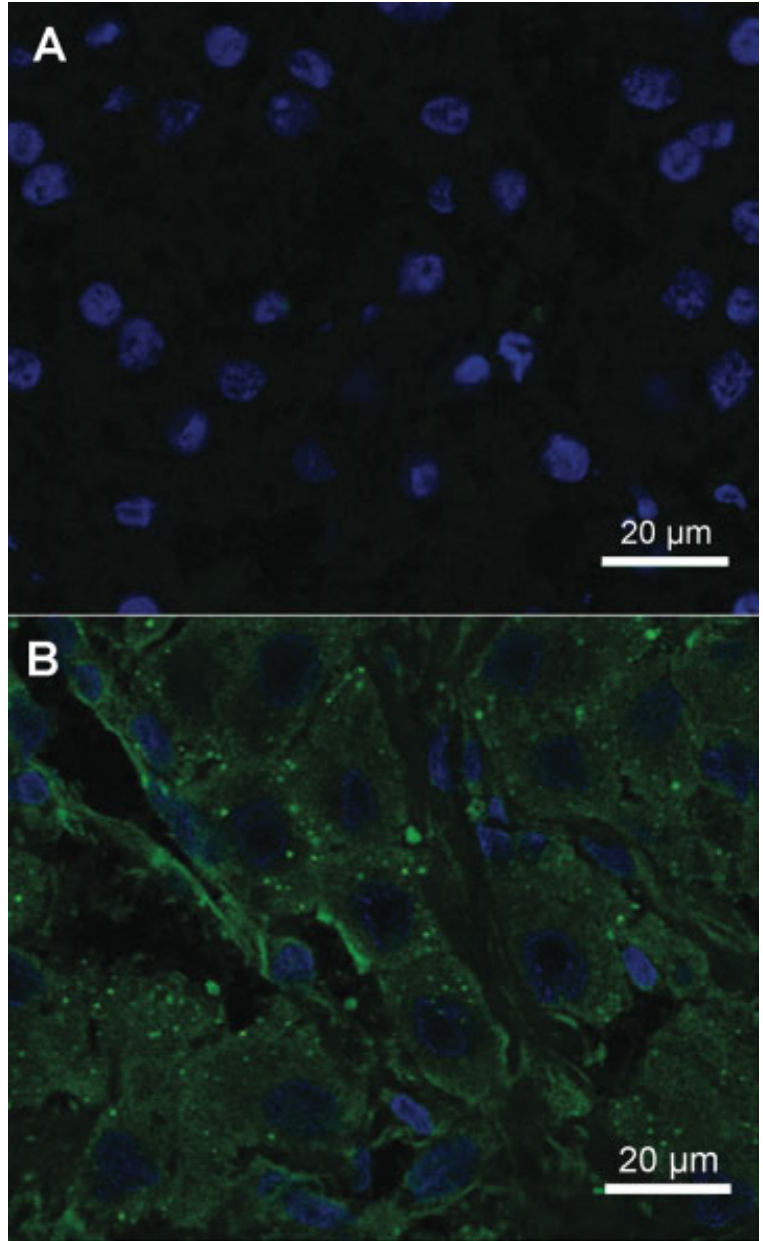

Fig. 3 Comparison of levels of PRKACA (green) in normal tissue (A) and FL-HCC tumor tissue (B) under confocal microscopy (nuclei are stained in blue). ${ }^{17}$ Originally published in: Honeyman JN et al. Detection of a recurrent DNAJB1-PRKACA chimeric transcript in fibrolamellar hepatocellular carcinoma. Science 2014; 343:1011. (C) 2014 American Association for the Advancement of Science. Reprinted with permission.

\section{Surgical Treatment}

Complete surgical resection remains the cornerstone of the treatment of FL-HCC. While small peripheral lesions may be treated with nonanatomic wedge resections with adequate margins, this may not be feasible as FL-HCC uncommonly presents in this manner. Many cases are diagnosed at advanced stages with larger tumors, ${ }^{8,9}$ and a major anatomic resection such as a right hepatectomy is often necessary. The extent of resection is based on a multitude of factors, including tumor location and proximity to vascular and ductal structures. Imaging, in the form of triple-phase CT or abdominal MRI, are imperative for preoperative planning as they provide crucial information on vascular and ductal anatomy. Notably, up to $85 \%$ of the liver parenchyma may be removed, as complete regeneration is often seen, even with postoperative chemotherapy.

Following a bilateral subcostal (or a right subcostal for patients with narrow costal margins) incision, the liver is mobilized by division of the right triangular and coronary

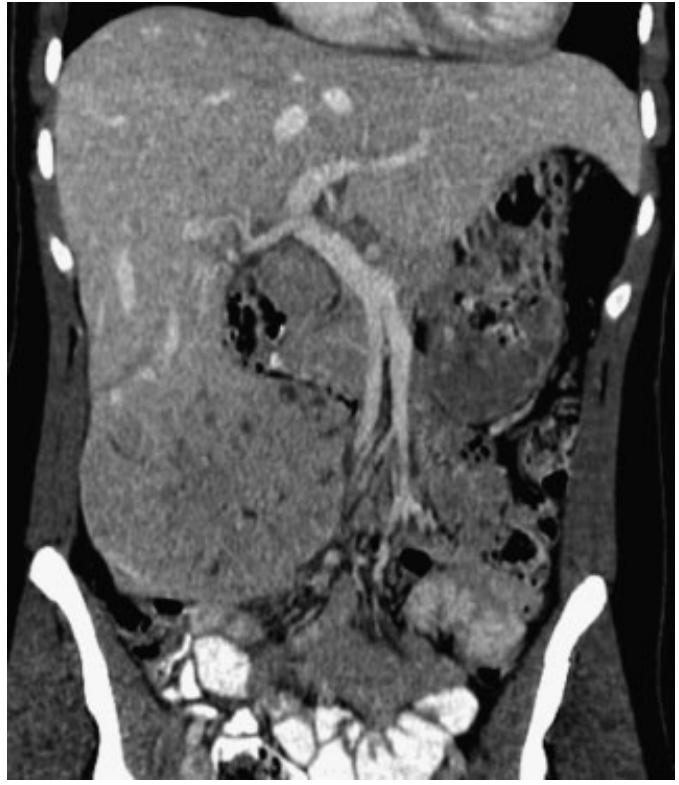

Fig. 4 Appearance of FL-HCC on CT scan. A heterogeneous, pedunculated mass is seen arising from the right lobe of the liver.

ligaments to the falciform ligament anteriorly and the inferior vena cava posteriorly. A combination of palpation and intraoperative ultrasound should be performed to identify multifocal disease. Once resectability is confirmed, the hepatoduodenal ligament is isolated with a looped vascular tape for subsequent Pringle maneuver. Vascular structures are controlled, either by extrahepatic vascular control or by hepatic pedicle ligation, prior to parenchymal division. Intraoperative management with anesthesia is key as maintenance of a lower central venous pressure minimizes blood loss during liver parenchymal transection. In rare cases, FL-HCC tumors may be focal lesions isolated to one segment that can be resected using anatomic segmentectomies. Preoperative, as well as intraoperative, imaging will aid in the dissection for control of arterial and portal inflow.

FL-HCC is associated with more favorable outcomes compared with conventional HCC when treated with surgical

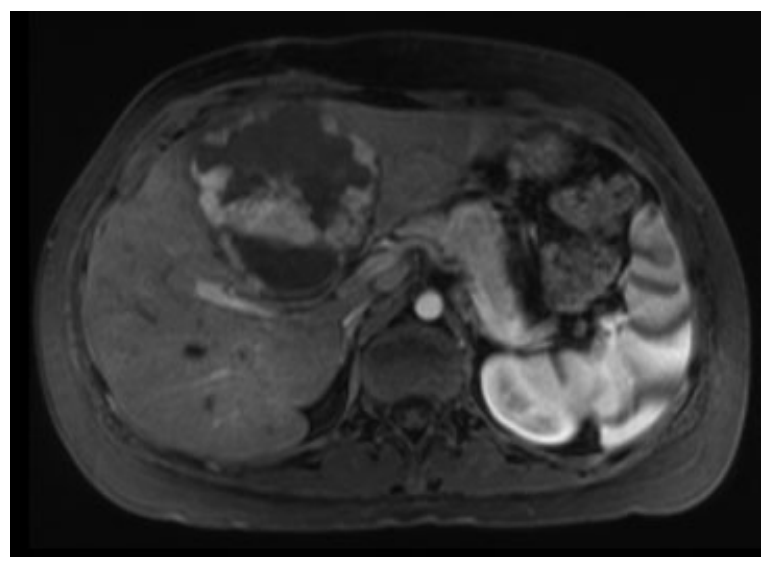

Fig. 5 Appearance of FL-HCC on MRI. This T1-weighted image shows a heterogeneously enhancing mass in segment IV. 
resection, with 5-year survival rates ranging from 58 to $82 \%{ }^{8,33}$ Resection of larger or even ruptured tumors ${ }^{34}$ is attempted more often for FL-HCC than for $\mathrm{HCC}^{8}$ due to its more favorable response to surgery. In fact, patients with complete resection are twice as likely to survive as those without successful surgical resection. ${ }^{30}$ Moreover, recurrence-free survival increases with $\mathrm{R} 0$ resection such that recurrence-free survival to 4 years may be predictive of disease-free survival. ${ }^{22}$ Recurrences, however, are common, with median recurrence-free survival rates ranging from 20 to 48 months. ${ }^{22,35}$ Common sites of recurrence include liver, regional lymph nodes, peritoneum, bones and lungs, with lymph nodes being the most frequent site of metachronous metastatic spread. ${ }^{36}$ Resection of these recurrences is thus becoming increasingly important, especially when they recur in the liver. ${ }^{22}$ In such cases, an anatomic segmentectomy may be appropriate.

In multiple studies, the presence of extrahepatic disease is a consistent, independent predictor of overall and recurrence-free survival. ${ }^{20,22,32}$ In particular, spread to regional lymph nodes is more common in FL-HCC than in conventional HCC. In a study by Mayo et al, the incidence of regional disease among patients with FL-HCC was more than $42.2 \%$ compared with $22.1 \%$ in conventional HCC. ${ }^{37}$ In one study, of 28 FL-HCC patients, $100 \% 5$-year survival was seen in lymph nodenegative patients compared with 45\% 5-year survival in lymph node-positive patients. ${ }^{35}$ Patients undergoing resection for FL-HCC may benefit from concomitant regional lymphadenectomy, as a recent study by the US National Cancer Institute's SEER Program reported superior survival rates in patients who underwent lymphadenectomy compared with those who did not (70\% vs. $57 \%){ }^{38}$

Transplantation for the treatment of HCC has long been a subject of debate, and guidelines such as the Milan criteria ${ }^{39}$ have been established. Specifically, orthotopic liver transplantation is employed as an alternative treatment when partial hepatectomy is otherwise contraindicated. ${ }^{40}$ Given its rare nature, FL-HCC infrequently requires transplantation and, thus, patients are not prioritized among those awaiting transplant. ${ }^{40}$ Of those few FL-HCC patients for whom transplantation is considered, the clinician must take into account the high rates of early lymph node metastasis, which may result in recurrence soon after liver transplant. ${ }^{41}$

\section{Nonoperative Management}

Various nonoperative modalities have been described as either adjuncts to surgery, or as down-staging mechanisms for patients with locally advanced, or metastatic disease with unresectable tumors. As patients with FL-HCC generally do not have a background history of cirrhosis or liver disease, they are thought to have the ability to tolerate higher doses of chemotherapy compared with conventional HCC patients. ${ }^{2,3}$

As a rare hepatic malignancy, FL-HCC currently has no standardized systemic drug treatments. Case reports and subgroup analyses of studies of liver cancers suggest possible activity for platinum-based agents, fluoropyrimidines and interferon- $\alpha$ (INF- $\alpha$ ); however, particular combinations of chemotherapeutic agents are not seen as standardized treatments throughout the literature. ${ }^{2,42}$ While there is no overall consensus in the chemotherapeutic management of FL-HCC, many case reports describe the use of gemcitabine/oxaliplatin (GEMOX) or 5-fluorouracil (5-FU) with INF- $\alpha$ and folinic acid/ 5 -FU/oxaliplatin (FOLFOX) with varying degrees of success in patients. $^{2,42,43}$ Radiation therapy is not typically used in primary management of FL-HCC, but has been described with recurrent and metastatic disease. ${ }^{4,27,42}$ A recent retrospective study has reported the use of intraoperative radiation therapy for distant nodal disease, which also warrants further research. ${ }^{32}$ With the new advances toward understanding the pathogenesis of FL-HCC, multi-institutional randomized clinical trials are ongoing, with one examining the role of estrogen suppression and MTOR inhibition and its potential therapeutic effects on FL-HCC (ClinicalTrials.gov Identifier: NCT01642186)*.

\section{Conclusion}

Liver cancers are uncommon in children and FL-HCC represents only a fraction of this population. As a rare tumor with limited established adjunctive therapies to surgical resection, further investigation is clearly warranted. Until then, surgery remains the only definitive treatment for this disease. Investigations in the tumor biology of FL-HCC, as well as research in improved methods of diagnosis and response to adjunctive therapies, will hopefully result in the development of effective nonoperative treatments to complement surgical resection.

\section{Acknowledgments}

The authors thank Dr. Anita P. Price from the Department of Radiology, Memorial Sloan Kettering Cancer Center, for assistance with radiographic imaging, and Rachel Belote from the Laboratory of Cellular Biophysics, Rockefeller University, for assistance with microscopy imaging.

\section{Conflict of Interest}

None.

\section{References}

1 Edmondson HA. Differential diagnosis of tumors and tumor-like lesions of liver in infancy and childhood. AMA J Dis Child 1956; 91(2):168-186

2 Fonseca GM, Varella AD, Coelho FF, Abe ES, Dumarco RB, Herman P. Downstaging and resection after neoadjuvant therapy for fibrolamellar hepatocellular carcinoma. World J Gastrointest Surg 2014;6(6):107-111

\footnotetext{
* This clinical trial (ClinicalTrials.gov Identifier: NCT01642186) is sponsored by Memorial Sloan Kettering Cancer Center in collaboration with: The Fibrolamellar Cancer Foundation; Dana-Farber Cancer Institute/Brigham and Women's Cancer Center and Massachusetts General Hospital Cancer Center; Johns Hopkins University; University of California, San Francisco; Abbott Laboratories; and Novartis Pharmaceuticals.
} 
3 Okur A, Eser EP, Yilmaz G, et al. Successful multimodal treatment for aggressive metastatic and recurrent fibrolamellar hepatocellular carcinoma in a child. J Pediatr Hematol Oncol 2014;36(5): e328-e332

4 Maniaci V, Davidson BR, Rolles K, et al. Fibrolamellar hepatocellular carcinoma: prolonged survival with multimodality therapy. Eur J Surg Oncol 2009;35(6):617-621

5 Torbenson M. Fibrolamellar carcinoma: 2012 update. Scientifica (Cairo) 2012;2012:743790

6 Paradis V. Histopathology of hepatocellular carcinoma. Recent Results Cancer Res 2013;190:21-32

7 Ganeshan D, Szklaruk J, Kundra V, Kaseb A, Rashid A, Elsayes KM. Imaging features of fibrolamellar hepatocellular carcinoma. AJR Am J Roentgenol 2014;202(3):544-552

8 Eggert T, McGlynn KA, Duffy A, Manns MP, Greten TF, Altekruse SF. Fibrolamellar hepatocellular carcinoma in the USA, 2000-2010: A detailed report on frequency, treatment and outcome based on the Surveillance, Epidemiology, and End Results database. United European Gastroenterol J 2013;1(5):351-357

9 Kaseb AO, Shama M, Sahin IH, et al. Prognostic indicators and treatment outcome in 94 cases of fibrolamellar hepatocellular carcinoma. Oncology 2013;85(4):197-203

10 Eggert T, McGlynn KA, Duffy A, Manns MP, Greten TF, Altekruse SF. Epidemiology of fibrolamellar hepatocellular carcinoma in the USA, 2000-10. Gut 2013;62(11):1667-1668

11 Ward SC, Huang J, Tickoo SK, Thung SN, Ladanyi M, Klimstra DS. Fibrolamellar carcinoma of the liver exhibits immunohistochemical evidence of both hepatocyte and bile duct differentiation. Mod Pathol 2010;23(9):1180-1190

12 Zen Y, Vara R, Portmann B, Hadzic N. Childhood hepatocellular carcinoma: a clinicopathological study of 12 cases with special reference to EpCAM. Histopathology 2014;64(5):671-682

13 Malouf GG, Job S, Paradis V, et al. Transcriptional profiling of pure fibrolamellar hepatocellular carcinoma reveals an endocrine signature. Hepatology 2014;59(6):2228-2237

14 Muramori K, Taguchi S, Taguchi T, et al. High aromatase activity and overexpression of epidermal growth factor receptor in fibrolamellar hepatocellular carcinoma in a child. J Pediatr Hematol Oncol 2011;33(5):e195-e197

15 Sulaiman RA, Geberhiwot T. Fibrolamellar hepatocellular carcinoma mimicking ornithine transcarbamylase deficiency. JIMD Rep 2014; [Epub ahead of print]

16 Riehle KJ, Yeh MM, Yu JJ, et al. mTORC1 and FGFR1 signaling in fibrolamellar hepatocellular carcinoma. Mod Pathol 2014; [Epub ahead of print]

17 Honeyman JN, Simon EP, Robine N, et al. Detection of a recurrent DNAJB1-PRKACA chimeric transcript in fibrolamellar hepatocellular carcinoma. Science 2014;343(6174):1010-1014

$18 \mathrm{Xu} \mathrm{L}$, Hazard FK, Zmoos AF, et al. Genomic analysis of fibrolamellar hepatocellular carcinoma. Hum Mol Genet 2014; [Epub ahead of print]

19 Ward SC, Waxman S. Fibrolamellar carcinoma: a review with focus on genetics and comparison to other malignant primary liver tumors. Semin Liver Dis 2011;31(1):61-70

20 Ang CS, Kelley RK, Choti MA, et al. Clinicopathologic characteristics and survival outcomes of patients with fibrolamellar carcinoma: data from the fibrolamellar carcinoma consortium. Gastrointest Cancer Res 2013;6(1):3-9

21 Kanai T, Takabayashi T, Kawano Y, Kuramochi S, Miyazawa N. A case of postoperative recurrence of fibrolamellar hepatocellular carcinoma with increased vitamin B12 binding capacity in a young Japanese female. Jpn J Clin Oncol 2004;34(6):346-351

22 Groeschl RT, Miura JT, Wong RK, et al. Multi-institutional analysis of recurrence and survival after hepatectomy for fibrolamellar carcinoma. J Surg Oncol 2014;110(4):412-415

23 Patonai A, Erdélyi-Belle B, Korompay A, et al. Molecular characteristics of fibrolamellar hepatocellular carcinoma. Pathol Oncol Res 2013;19(1):63-70
24 Liu S, Wah Chan K, Tong J, Wang Y, Wang B, Qiao L. PET-CT scan is a valuable modality in the diagnosis of fibrolamellar hepatocellular carcinoma: a case report and a summary of recent literature. QJM 2011;104(6):477-483

25 Sun L, Wu H, Guan YS. Positron emission tomography/computer tomography: challenge to conventional imaging modalities in evaluating primary and metastatic liver malignancies. World J Gastroenterol 2007;13(20):2775-2783

26 Do RK, McErlean A, Ang CS, DeMatteo RP, Abou-Alfa GK. CT and MRI of primary and metastatic fibrolamellar carcinoma: a case series of 37 patients. Br J Radiol 2014;87(1040):20140024

27 G Peacock J, A Call J, R Olivier KRadiotherapy for metastatic fibrolamellar hepatocellular carcinoma. Rare Tumors 2013;5(3):e28

28 Trojan J, Schroeder O, Raedle J, et al. Fluorine-18 FDG positron emission tomography for imaging of hepatocellular carcinoma. Am J Gastroenterol 1999;94(11):3314-3319

29 von Falck C, Rodt T, Shin HO, Knapp WH, Galanski M. F-18 FDG PET imaging of fibrolamellar hepatocellular carcinoma. Clin Nucl Med 2008;33(9):633-634

30 Weeda VB, Murawski M, McCabe AJ, et al. Fibrolamellar variant of hepatocellular carcinoma does not have a better survival than conventional hepatocellular carcinoma-results and treatment recommendations from the Childhood Liver Tumour Strategy Group (SIOPEL) experience. Eur J Cancer 2013;49(12):2698-2704

31 Katzenstein HM, Krailo MD, Malogolowkin MH, et al. Fibrolamellar hepatocellular carcinoma in children and adolescents. Cancer 2003;97(8):2006-2012

32 Darcy DG, Malek M. M., Kobos, R., Klimstra, D.S., DeMattero, R. LaQuaglia, M.P. Prognostic factors in fibrolamellar hepatocellular carcinoma in young people. J Pediatr Surg 2014; In press DOI

33 Mavros MN, Mayo SC, Hyder O, Pawlik TM. A systematic review: treatment and prognosis of patients with fibrolamellar hepatocellular carcinoma. J Am Coll Surg 2012;215(6):820-830

34 Minutolo V, Licciardello A, Arena M, Minutolo O, Lanteri R, Arena G. Surgical resection of ruptured fibrolamellar hepatocellular carcinoma. Case Rep Surg 2013;2013:679565

35 Stipa F, Yoon SS, Liau KH, et al. Outcome of patients with fibrolamellar hepatocellular carcinoma. Cancer 2006;106(6):1331-1338

36 Wojcicki M, Lubikowski J, Post M, Chmurowicz T, WiechowskaKozlowska A, Krawczyk M. Aggressive surgical management of recurrent lymph node and pancreatic head metastases of resected fibrolamellar hepatocellular carcinoma: a case report. JOP 2012; 13(5):529-532

37 Mayo SC, Mavros MN, Nathan H, et al. Treatment and prognosis of patients with fibrolamellar hepatocellular carcinoma: a national perspective. J Am Coll Surg 2014;218(2):196-205

38 McAteer JP, Goldin AB, Healey PJ, Gow KW. Hepatocellular carcinoma in children: epidemiology and the impact of regional lymphadenectomy on surgical outcomes. J Pediatr Surg 2013; 48(11):2194-2201

39 Mazzaferro V, Regalia E, Doci R, et al. Liver transplantation for the treatment of small hepatocellular carcinomas in patients with cirrhosis. N Engl J Med 1996;334(11):693-699

40 Grossman EJ, Millis JM. Liver transplantation for non-hepatocellular carcinoma malignancy: Indications, limitations, and analysis of the current literature. Liver Transpl 2010;16(8):930-942

41 Hackl C, Schlitt HJ, Kirchner GI, Knoppke B, Loss M. Liver transplantation for malignancy: current treatment strategies and future perspectives. World J Gastroenterol 2014;20(18): 5331-5344

42 Fakih M. A case of fibrolamellar cancer with a palliative response and minor radiographic regression with erlotinib and bevacizumab combination therapy. Am J Ther 2013: doi: 10.1097/ MJT.0b013e3182840fa6

43 Gras P, Truant S, Boige V, et al. Prolonged complete response after GEMOX chemotherapy in a patient with advanced fibrolamellar hepatocellular carcinoma. Case Rep Oncol 2012;5(1):169-172 